\title{
Ophthalmological phenomena
}

Keywords: phenomenon, phenomena, ocular, ophthalmological

\section{Opinion}

The phenomenon is defined as "an event or fact or situation that is observed to exist or happen", or "something that exists and manifests itself and can be seen or perceived with sensations". It is often unusual or interesting. The term is originated from the word "phainomenon" in Greek with the meaning of "thing appearing to view" and entered into Latin alphabet. Its plural form is phenomena., ${ }^{1,2}$ Phenomenon in philosophy has been defined as "any object, fact, or occurrence perceived or observed". ${ }^{3}$ Scientists uses it to describe any event or fact that could be observed, amazing or not. In medical literature, it is often used in the meaning of a symptom; an occurrence of any sort, whether ordinary or extraordinary, in relation to a disease. ${ }^{4}$ The term "phenomenon" can sometimes be miscalled equally to the terms "sign or reflex". However, terms "syndrome and disease" are not often confused with the phenomenon. Reflex is defined as " an involuntary physical reaction or automatic response from the nerve impulse to a stimulus. ${ }^{5-7}$ On the other hand, the term in medicine "sign" is any abnormal, observable evidence or physical phenomenon which often indicates dysfunction or disease. It is usually detected by the physician even if the patient cannot observe and experience it. ${ }^{8,9}$ Regarding phenomena in ophthalmology, we fund just a publication by Sharma et al., ${ }^{10}$ in the web. Here, following the search of the term in major academical and scientific databases and indexes, we tried to present in alphabetical order, some common phenomena in ophthalmology. Some of these_are actual phenomena that we have known already before but are called differently.

\section{A-V phenomenon}

If the angle of strabismus is greater in upgaze and lower in downgaze, the status of the eyes cause the letter $\langle\mathrm{V}\rangle$ appearance. Conversely, if the angle of strabismus is lower in upgaze and greater in downgaze, the status of the eyes forms to the Greek letter " $\Lambda$ ". However, the nomenclature " $A$ " letter instead of " $\Lambda$ " in English spelling of this phenomenon has been used. "V" phenomenon often mainly seen in the bilateral hyperfunction of the lower oblique muscles, while as "A" phenomenon occurs in the bilateral hyperfunction of the upper oblique muscles. ${ }^{11}$

\section{Argyll-Robertson phenomenon}

The phenomenon is defined as no or poorly reacting irregular and narrow pupils to light but reactive to near vision when looking at a target within near distance ("light-near dissociation"). It can be observed neurosyphilis, diabetes, multiple sclerosis and dorsal midbrain syndrome. ${ }^{12}$

\section{Aschner-Dagnini phenomenon (Oculocardiac reflex, trigemino-vagal reflex)}

It is defined as the episode of dramatic bradycardia following pressure on globe or traction/manipulation of extra-ocular muscles in ocular surgery due to the vagal response stimulated by the connection between the trigeminal and vagal nerves. ${ }^{13}$

\author{
Volume 8 Issue 4 - 2018

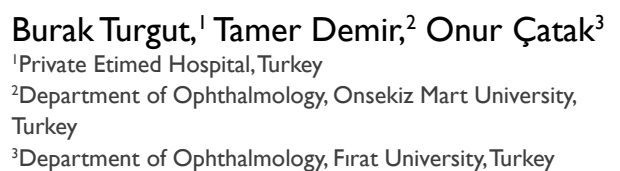

Correspondence: Burak Turgut, Private Etimed Hospital, Ophthalmology Clinic, Elvan Mah, 1934. Sok. No: 4 Etimesgut/Ankara, Turkey, Tel +90-3122-9306-06, Email drburakturgut@gmail.com

Received: May 28, 2018| Published: July 03, 2018

\section{Bell's phenomenon}

This protective reflex is described as the upward and slightly outward movement of the globe during the eyelid closure to avoid corneal exposure. ${ }^{14,15}$

\section{Blue-field entoptic phenomenon (Scheerer's phenomenon)}

It is to be perceived of the leukocytes moving in the retinal capillaries of the own retina of the individual. This phenomenon is not affected by providing that the reaching of blue light to the retina is enough while as media opacities does not prevent for establishing this phenomenon. The person experiences tiny bright dots which rapidly move along squiggly lines in the visual field. ${ }^{16}$

\section{Crowding phenomenon}

It shows that neighboring targets interfere as proximity increases and is described as "having better visual acuity for single optotypes (Sheridan Gardiner) than multiple optotypes (Snellen)". Especially, amblyopic patients usually present this phenomenon. ${ }^{17}$

\section{Doll's eye phenomenon (Oculocephalic reflex)}

It is the movement in the opposite direction of the eyes to the head movement seen by the observer in order to keep the eyes stable in space and to stabilize the visual world on the retina. As the head is turned rapidly to one side, the globes conjugately turn to the direction opposite to the head's movement. The loss of this reflex implies the dysfunction of brainstem or oculomotor nerves. ${ }^{18,19}$

\section{Ewart phenomenon}

It is a type of synkinesis due to aberrant nerve degeneration and is defined as the elevation of a ptotic eyelid on swallowing. ${ }^{20}$

\section{Fish-eggs phenomenon}

This phenomenon is to see multiple globules, droplets or bubbles belonging to emulsified silicone or fluoro-silicone oil in the vitreous cavity or anterior chamber due to dispersion and diffusion of a tamponade. ${ }^{21}$ 


\section{Heavy-eye phenomenon}

It includes unilateral high myopia, amblyopia, progressive exotropia, hypotropia, reduced upgaze, pseudoptosis and increased axial length. It is due to compression of the lateral rectus muscle against the lateral orbital wall by the enlarged myopic globe. ${ }^{22}$

\section{Haidinger-brush phenomenon}

It is the entoptic phenomena in which two subtle bowties, a blue one, and a yellow one, overlapping each other at 90 degrees or hourglass-shaped/radial pattern displays appear during the view of a field having the blue light (computer screen and sky). This phenomenon is attributed to the dichroism of the xanthophyll pigment of the macula and response of the eyes to polarized light and to the preferential absorption of blue polarized light. If the light is all blue, it seems like a dark shadow while as if the light is full spectrum, it is observed as yellow color. ${ }^{23}$

\section{Heimann-Bielschowsky phenomenon}

It is an asymptomatic monocular slow, pendular, mostly vertical nystagmus or oscillation movement with variable amplitude. It is considered that a disruption in vertical fusional vergence or monocular visual stabilization system due to monocular severe visual loss plays role in its pathogenesis. ${ }^{24}$

\section{Hemi-field slide phenomenon}

It presents the instability of ocular alignment and the loss of corresponding retinal points in the two eyes, allowing an underlying phoria to manifest as a tropia. This phenomenon is a rare sensory phenomenon presenting with intermittent and transient binocular diplopia with bitemporal hemianopia without an oculomotor paresis. It can occur in the patients with optic chiasm lesion like pituitary adenoma. ${ }^{25}$

\section{Inverse Bell's phenomenon}

In this phenomenon, the eye moves downward instead of upward during eyelid closure. It may be seen in the normal subjects, the cases with Bell's palsy or conjunctival scarring. ${ }^{14,15}$

\section{Jack-in-the-box phenomenon}

It is described as the ring scotoma and the sudden disappearance and sharp reappearance of the objects due to the prismatic effect of a positive lens at the edge of a spectacle frame. An object seeing in the peripheral visual field is perceived blurry at the region of the side of the spectacle frame. To see this object clearly, the person tries to move the head to the object. However, when the heat is turned towards the object, it exists in the scotoma area and disappears again. Then, the person turns the head further and the object enters the visible area and it reappears again clearly and sharply. ${ }^{26}$

\section{Leash phenomenon}

It is described as up and down shoots and globe retraction due to co-innervation of the horizontal and vertical rectus muscles or slippage of the lateral rectus muscle above or below the eye. ${ }^{27}$

\section{Marcus Gunn jaw-winking phenomenon}

In this uncommon congenital synkinetic (aberrant regeneration) phenomenon, unilaterally ptotic upper eyelid elevates concomitantly and involuntarily with various mandible movements due to cross innervations between oculomotor nerve and mandibular branch of trigeminal nerve. ${ }^{28}$

\section{Mizuo-Nakamura phenomenon}

This phenomenon is characterized by a change in the color of the fundus from red in prolonged dark-adaptation to a golden yellow metallic sheen with light exposure. The color of the fundus reflex in the light-adapted state may be also as golden-yellow, yellow-white or gray-white. The phenomenon has been described in Oguchi disease (an autosomal recessive stationary night blindness) and X-linked cone dystrophy. It has been hypothesized that excessive extracellular potassium in the retina might be related to this phenomenon. ${ }^{29}$

\section{Oculo-digital phenomenon (Franceschetti-Leber phenomenon)}

It is the sign of congenital or early acquired blindness and includes the manipulating the eyelid, poking, pressing, and rubbing of the eye by affected child's own knuckle or finger. ${ }^{30}$

\section{Paradoxical constriction of pupils to darkness (Flynn phenomenon)}

In normal condition, the pupils dilate bilaterally as a response to darkness. However, in this phenomenon, the pupils constrict bilaterally paradoxically in darkness. It has been reported in the patients with Leber's congenital amaurosis, congenital stationary night-blindness, congenital nystagmus or achromatopsia, strabismus, amblyopia, bilateral optic neuritis, and dominant optic atrophy. Although the exact pathogenic mechanism of this phenomenon is not clearly known, it is considered that the rods send a "bleaching signal" after a strong illumination and immediate transfer to darkness, this signal over-rules the cone malfunctioning, as paradoxical pupillary constriction. $^{31}$

\section{Pseudo-Graefe phenomenon (Fuch's sign)}

This synkinetic phenomenon includes the paradoxical upper eyelid elevation or retraction and improvement of ptosis on adduction or downward gaze observed during usual recovery from oculomotor nerve paralysis (aberrant regeneration). Pseudo-Graefe phenomenon may occur congenitally or acquirable following oculomotor paralysis and intracranial aneurysms, traumas and tumors. . $^{32,33}$

\section{Pulfrich's phenomenon}

It is defined as a stereoscopic illusion and perception defect of movement in two eyes due to differential signal timings to the cortex and the temporal disparity between the eyes. Because a decreased luminance increases retinal processing time, the information reaching the cortex delays relative to the normal eye. This delay in time between the two eyes is interpreted by the cortex as a separation in space. The movement is always perceived as toward the diseased eye, counterclockwise and the other eye clockwise. Pulfrich phenomenon can occur in ocular injury, ischemia, or glaucomatous optic nerve damage and resolved optic neuritis. ${ }^{34}$

\section{Purkinje's entoptic phenomenon}

It is the ability to visualize the shadow images of person's own endogenous ocular structures or substances such as retinal vasculature (Purkinje tree) and opacities in vitreous when the illumination was applied on a closed eyelid or the anterior segment. The term 
'entoptic" origins from the Greek words 'inside' and 'light'. Entoptic phenomena were first described by Johann Purkinje. It may be distinguished from a real image with the lack of tracking with eye or retinal movement. ${ }^{35,36}$

\section{Riddoch's phenomenon (Statokinetic dissociation)}

It is an ophthalmological phenomenon in which the person can visually perceive only moving (kinetic) objects but not static objects and the color or shape of these objects. It is observed in the patients with lesions in the anterior visual pathways or the occipital lobe. ${ }^{37}$

\section{Steal phenomenon}

This phenomenon is defined as the reversion of the blood flow in the ophthalmic artery to supply the ipsilateral brain in patients with incomplete blood circulation in the circle of Willis and internal carotid artery occlusion. It results in ocular ischemia. ${ }^{38}$

\section{Sunset eye phenomenon (setting sun phenomenon)}

This phenomenon is an earlier finding of the raised intracranial pressures or hydrocephalus resulting from upward-gaze paresis in infants. In this phenomenon, the eyes are at the position moved downward, the sclera can see between the upper eyelid and the iris, and lower part of the pupil has been covered by the lower eyelid. Although its pathogenesis is not well understood, it has been considered that it may be related to aqueduct distention with compression of periaqueductal structures secondary to increased intracranial pressure. ${ }^{39}$

\section{Tyndall phenomenon}

It shows the breakdown of the blood-aqueous barrier and increased protein concentration into the aqueous humor. It often seems as scattered small particles and flare and it causes a scattered beam light in blue color in anterior chamber. ${ }^{40}$

\section{Troxler's phenomenon (troxler's fading)}

It is optical illusion in visual perception. Approximately 2030 seconds following the fixation of the eyes to a point (extended stabilization of gaze), a stimulus in peripheral vision (away from the fixation point) fades and disappears. The phenomenon will be more significant when the stimulus with small or low contrast or blurred stimulus is used. It is considered that it might result from a timedependent visual neuronal adaptation. ${ }^{41}$

\section{Uthoff's phenomenon}

It is described as transiently decreased visual acuity on exertion or by an increase in body temperature (hot conditions such as fever, hot bath or vigorous exercise) in the cases with optic neuritis secondary multiple sclerosis. It is considered that increased temperature causes the prolonged inactivation of voltage-gated sodium channels and a transient blockage of nerve conduction in demyelinated nerve fibers. ${ }^{42}$

\section{Visual phenomena}

It means any visual perception including a form of the positive scotoma (photopsia, sparkles, flashes, flickering/shimmering/ bright/multicoloured or zig-zag shaped lights, geometric forms and scintillating/migratory scotomas, as seen in migraine and seizure or negative form (visual field defects, shady/blurry/foggy, hazy, patchy/ dimly vision) in as occurred in ischemic visual loss. ${ }^{43}$

\section{Wipe-out phenomenon (Snuff syndrome)}

It is the development of severe visual loss without an obvious cause which is known after glaucoma surgery. It is thought that a direct damage to the optic nerve due to local anesthesia (optic nerve sheath hematoma or injected volume) might cause this uncommon complication. ${ }^{44}$

\section{Acknowledgments}

None.

\section{Conflict of interest}

The author declares that there is no conflict of interest regarding the publication of this paper.

\section{Authorship contributions}

All authors have contributed to the concept and design, data collection, literature Search in the work and writing of the manuscript.

\section{References}

1. https://dictionary.cambridge.org/dictionary/english/phenomenon

2. https://en.oxforddictionaries.com/definition/phenomenon

3. https://www.britannica.com/topic/phenomenon-philosophy

4. https://medical-dictionary.thefreedictionary.com/phenomenon

5. http://www.dictionary.com/browse/reflex

6. https://dictionary.cambridge.org/dictionary/english/reflex

7. https://en.oxforddictionaries.com/definition/reflex

8. https://medical-dictionary.thefreedictionary.com/sign

9. https://en.oxforddictionaries.com/definition/sign

10. Sharma RL, Sharma K, Sharma M, et al. Clinical signs and phenomenon in ophthalmology. DOS Times. 2016;83-84.

11. Straight SM, Bahl RS. A- and V-Pattern Strabismus. In: Traboulsi E, Utz V, editors. Practical Management of Pediatric Ocular Disorders and Strabismus. New York, NY: Springer; 2016. p. 583-592.

12. Abramson JL, Teitelbaum MH. The Argyll Robertson Phenomenon in Multiple Sclerosis. Am J Ophthalmol. 1933;16(8):676-682.

13. Aschner B. Ueber einen bisher noch nicht heschriebenen reflex vom auge auf k.reislauf und atmung: Verschwinden des Radialispulses bei Druck auf das Auge. Wien Kiln Wochenscr 1908;21:1529-1530.

14. Schiefer U, Wilhelm H, Hart W. Clinical Neuro-Ophthalmology. A Practical Guide. Springer-Verlag Berlin Heidelberg, 2007.

15. Shitole S, Jakkal T, Khaire B. Inverse Bell's Phenomenon: Rare Ophthalmic Finding Following Ptosis Surgery. J Clin Diagn Res. 2015;9(3):ND01-ND02.

16. Jan Dirk Blom. A Dictionary of Hallucinations. New York: SpringerVerlag; 2010, p. 59-80.

17. Stuart JA, Burian HM. A study of separation difficulty its relationship to visual acuity in normal and amblyopic eyes. Am J Ophthalmol. $1962 ; 53: 471-477$.

18. Ropper AH, Brown HB. Oculocephalic reflexes. In: M Victor, AL Ropper, Editors. Adams + Victor's Principles of Neurology, 7th ed. New York: McGraw-Hill; 2001, p. 380-381. 
19. Gordon N. The Doll's eye phenomenon. Dev Med Child Neurol. 1977;19:695-696.

20. Larner AJ. Synkinesia, synkinesis. In: A Dictionary of Neurological Signs. Fourth edition. Springer International Publishing, Cham, 2016, p. 289-310

21. Morescalchi F, Costagliola C, Duse S, et al. Heavy silicone oil and intraocular inflammation. Biomed Res Int. 2014;2014:574825.

22. Taylor R, Whale K, Raines M. The heavy eye phenomenon: orthoptic and ophthalmic characteristics. Ger J Ophthalmol. 1995;4(4):252-255.

23. Myrowitz E. A recent explanation of Haidinger's brushes and their clinical use. Am J Optom Physiol Opt. 1979;56(5):305-308.

24. Surachatkumtonekul T, Pamonvaechavan P. Unilateral vertical nystagmus: The Heimann-Bielschowsky phenomenon. J Med Assoc Thai. 2009;92(3):373-376.

25. Peragallo JH, Bialer OY, Pineles SL, et al. Hemifield slide phenomenon as a result of heteronymous hemianopia. Neuro-Ophthalmology. 2014;38(2):82-87.

26. Walsh G, Pearce EI. Is the spectacle lens jack-in-the-box phenomenon really due to the lenses? Ophthalmic Physiol Opt. 2006;26(1):116-119.

27. Anvari F, Hatef E, Mohammadi SF, et al. Duane's retraction syndrome, a case series from Iran. Int Ophthalmol. 2008;28(4):275-280.

28. Sundareswaran S, Nipun CA, Kumar V. Jaw-winking phenomenon: Report of a case with review of literature. Indian J Dent Res. 2015;26(3):320323.

29. Kumar V, Goel N, Bhaskaran UK, et al. Mizuo-Nakamura phenomenon in cone-rod dystrophy. Clin Exp Optom. 2017;100(4):388-391.

30. Mansour AM, Reinecke RD. The pop eye phenomenon: an extreme form of the oculodigital phenomenon. J Clin Neuroophthalmol. 1985;5(4):281282 .

31. Ben Simon GJ, Abraham FA, Melamed S. Pingelapese achromatopsia: correlation between paradoxical pupillary response and clinical features. Br J Ophthalmol. 2004;88(2):223-225.

32. Martorina M, Porté E. Pseudo-Graefe's sign: a manifestation of aberrant regeneration of the fourth cranial nerve? Graefes Arch Clin Exp Ophthalmol. 1993;231(2):76-78.
33. Gaddipati RV, Meyer DR. Eyelid retraction, lid lag, lagophthalmos, and von Graefe's sign quantifying the eyelid features of Graves' ophthalmopathy. Ophthalmology. 2008;115(6):1083-1088.

34. Farr J, McGarva E, Bijvank JN, et al. The Pulfrich phenomenon: Practical implications of the assessment of cases and effectiveness of treatment. Neuro-Ophthalmology. 2018.

35. Mark HH. The entoptic view of the retinal vessels. Acta Ophthalmol. 2014;92(3):e237-240.

36. Dolecek RL, de Launay J. Entoptic mapping of the Purkinje blue arcs. $J$ Opt Soc Am. 1945;35(10):676-680.

37. Hayashi R, Yamaguchi S, Narimatsu T, et al. Statokinetic dissociation (Riddoch phenomenon) in a patient with homonymous hemianopsia as the first sign of posterior cortical atrophy. Case Rep Neurol. 2017;9(3):256260 .

38. Costa VP, Kuzniec S, Molnar LJ, et al. Collateral blood supply through the ophthalmic artery. A steal phenomenon analyzed by color Doppler imaging. Ophthalmology. 1998;105(4):689-693.

39. Yoshikawa H. Benign Setting sun phenomenon in full-term infants. $J$ Child Neurol. 2003;18(6):424-425.

40. Lou L. Troxler effect with dichoptic stimulus presentations: Evidence for binocular inhibitory summation and interocular suppression. Vision Res. 2008;48(14):1514-1521.

41. Tyndall J. On the blue of the sky, the polarization of the skylight, and on the polarization of light by cloudy matter generally. Philos Mag J. $1869 ; 37: 384-404$.

42. Opara JA, Brola W, Wylegala A, et al. Uhthoff's phenomenon 125 years later-what do we know today? J Med Life. 2016;9(1):101-105.

43. Adamczyk DT. Visual phenomena, disturbances, and hallucinations. Optom Clin. 1996;5(3-4):33-52.

44. Abdelrahman AM, ElSaied HMA, Allam RSHM, et al. Wipe-out after subscleral trabeculectomy in advanced glaucoma patients. Delta Journal of Ophthalmology. 2017;18(2):94-98. 